\title{
Traduire
}

Une autre perspective sur rit traduction

Revue française de la traduction

$243 \mid 2020$

Les arts du spectacle

\section{Les langages de la danse}

Entretien croisé avec Florence Corin, Baptiste Andrien (éditeurs) et Aude Fondard (traductrice)

Baptiste Andrien, Florence Corin, Aude Fondard et Émilie Syssau

\section{Q OpenEdition}

12 Journals

Édition électronique

URL : http://journals.openedition.org/traduire/2171

DOI : $10.4000 /$ traduire. 2171

ISSN : 2272-9992

Éditeur

Société française des traducteurs

Édition imprimée

Date de publication : 15 décembre 2020

Pagination : 65-76

ISSN : 0395-773X

\section{Référence électronique}

Baptiste Andrien, Florence Corin, Aude Fondard et Émilie Syssau, "Les langages de la danse »,

Traduire [En ligne], 243 | 2020, mis en ligne le 15 décembre 2020, consulté le 31 décembre 2020. URL : http://journals.openedition.org/traduire/2171 ; DOI : https://doi.org/10.4000/traduire.2171 


\section{Les langages de la danse}

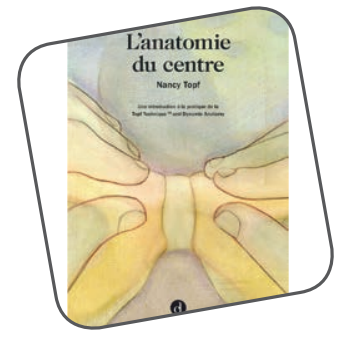

Entretien croisé avec Florence Corin, Baptiste Andrien (éditeurs) et Aude Fondard (traductrice)

Les éditions Contredanse, créées en 1990, répondent-elles au constat d'un manque dans le monde de la danse?

Florence Corin: L'association Contredanse a vu le jour à l'initiative de Patricia Kuypers, qui souhaitait élargir le paysage de la danse à Bruxelles, notamment en invitant des chorégraphes et des artistes qui n'y avaient jamais enseigné, en faisant découvrir des pratiques qu'on connaissait peu en Belgique, en mettant en place une bibliothèque de référence et en créant un médium qui fasse le lien au sein de la communauté dansante. La revue Nouvelles de danse est née quelques années après la création de l'association; c'était un petit cahier qui réunissait des informations pratiques (cours, stages et auditions) et du contenu, sous la forme d'un dossier thématique. Patricia a toujours voulu donner la parole aux danseurs et aux chorégraphes, faire se côtoyer des textes historiques et d'autres liés à la pratique, et stimuler l'écriture du monde de la danse. C'est aussi la période où, ayant rencontré Steve Paxton et le Contact Improvisation, elle a voulu partager sa découverte. La revue américaine Contact Quarterly' représentait alors une ressource importante pour 
les éditions Contredanse et les danseurs en général. Ceux qui ne lisaient pas l'anglais pouvaient avoir accès à ces textes par le biais des traductions.

Au fil des années, la revue a évolué en fonction des envies internes, des rencontres et des suggestions. Par exemple, le numéro On the Edge - Créateurs de l'imprévu² a été réalisé sur une proposition d'Agnès Benoît de mener et de traduire des interviews d'improvisateurs. Pour chaque numéro, on constituait une petite équipe rédactionnelle de spécialistes, on épluchait les ressources de notre centre de documentation, on se souvenait des textes qui nous avaient marqués, on s'interrogeait sur ce qu'il était pertinent ou non de traduire, et on sollicitait les auteurs. A ensuite émergé la volonté d'avoir des temporalités plus longues: le temps d'écriture était parfois trop court par rapport aux thématiques, d'autant plus si les auteurs n'étaient pas francophones. Nous sommes donc passés à une publication annuelle et parfois à des traductions plus conséquentes.

Baptiste Andrien: Nous avons aussi lancé un nouveau format de collaboration en nous rapprochant d'un artiste en particulier. Ces projets demandent un temps assez conséquent de développement, en termes de nature, de contenu et de forme. Il y a eu ceux avec Steve Paxton ${ }^{3}$ et Anna Halprin ${ }^{4}$, et nous menons actuellement ce travail avec Lisa Nelson.

Vous publiez donc en français des ouvrages originaux qui sont le résultat d'une traduction, mais qui n'existent pas forcément dans le domaine anglophone.

BA: C'est tout nouveau. Nous franchissons maintenant le pas de publier en anglais. Si Contredanse a pour mission de valoriser les écrits en français, ou en tout cas de prendre en charge des traductions afin de produire des documents pour le monde francophone, nous dérogeons un peu à cette règle pour mettre en valeur le travail de collaboration avec un

2. Nouvelles de danse, $n^{\circ} 32-33,2009$.

3. Steve Paxton, Baptiste Andrien et Florence Corin, Material for the Spine, une étude du mouvement / a movement study, traduit et sous-titré par Denise Luccioni, 2008.

4. Anna Halprin, Florence Corin et Baptiste Andrien, Dancing Life / Danser la vie, traduit par Denise Luccioni, 2009. 
artiste. L'ouvrage de Steve Paxton, Gravity, est ainsi d'abord paru en anglais ${ }^{5}$ avant qu'on le traduise en français ${ }^{6}$.

FC: En parallèle de ces projets de collaboration sur le long terme, nous aimons revenir aux textes qui sont des jalons dans la culture de la danse, en traduisant des écrits fondateurs, comme Sentir, ressentir et agir de Bonnie Bainbridge Cohen $^{7}$, Le Corps pensant de Mabel Todd ${ }^{8}$, Espaces dynamiques de Rudolf Laban ${ }^{9}$ ou notre dernière publication, L'Anatomie du centre de Nancy Topf ${ }^{10}$, traduite par Aude ici présente. Ce sont des textes dont la traduction nous paraît nécessaire pour continuer à élargir la culture de la danse en français.

En danse, il n'existe pas de système de notation unique comme en musique. Est-il plus facile de décrire le mouvement que de le noter? Y a-t-il une double traduction, à savoir traduire le mouvement en mots, puis traduire ces mots dans une nouvelle langue?

FC: Tout passage d'un médium à un autre est une traduction. Dans le ton et la manière, les possibilités de traduire l'expérience sont multiples. En fonction de l'enjeu, de ce qu'on veut traduire de la danse ou d'un mouvement, on peut arriver à des formes très différentes.

Aude Fondard: Le passage d'une langue à l'autre se fait par étapes, des étapes qui se retrouvent dans le corps et l'esprit d'un e interprète. Quand, en cours de danse, on me dit «un pas à droite, un pas à gauche», une traduction sensorielle se fait. Ca me dérange de dire «traduire le mouvement en mots, puis traduire la traduction du mouvement», car traduire le mouvement en mots est valable aussi en studio, quand des personnes d'une même langue vivent le partage de la création. Pour ma part, j'ai appris la danse en anglais; quand je suis revenue en France, mon cerveau ne comprenait pas les indications qu'on me donnait, parce que j'avais l'habitude d'entendre d'autres mots. Mon corps reproduisait, et alors

5. Steve Paxton, Gravity, 2018.

6. Steve Paxton, La gravité, traduit par Denise Luccioni, 2018.

7. Nouvelles de danse, $n^{\circ} 50$ : «Sentir, ressentir et agir», traduit par Madie Boucon, 2002.

8. Mabel E.Todd, Le Corps pensant, traduit par Élise Argaud et Denise Luccioni, 2012.

9. Rudolf Laban, Espace Dynamique - Textes inédits, 2003.

10. Nancy Topf, L'Anatomie du centre, traduit par Aude Fondard, 2020. 
seulement mon cerveau intégrait. Pour la traduction d'écrit à écrit, il se passe la même chose. Le livre de Nancy Topf décrit des exercices pratiques, que j'ai faits pour bien les traduire. En danse, la première traduction passe par le faire.

BA: À trois, nous avons évalué chaque proposition de traduction, sa pertinence par rapport à l'original et son registre ou niveau d'adresse.

AF : L'ouvrage L'Anatomie du centre est écrit par deux autrices, une pour la partie historique (Melinda Buckwalter), l'autre pour la technique Topf en tant que telle (Nancy Topf). II y a donc deux écritures distinctes, qui seront nécessairement traitées différemment dans la traduction. Par ailleurs, Baptiste et Florence avaient à cœur que certains mots soient exactement les mêmes qu'en anglais. Pour moi, le titre du chapitre "The long journey of the psoas» véhiculait un certain souffle en plus des éléments anatomiques. Je trouvais important de ne pas traduire "le long voyage du psoas», mais de parler de "périple». Ce n'était pas une question de registre, plutôt de renvoi intrinsèque à la notion de difficulté. Les termes «épopée» ou «odyssée» auraient été beaucoup trop éloignés, alors que "périple du psoas» offrait une assonance qui me semblait adéquate. Cette expression peut paraitre trop littéraire: on ne parlerait pas de "périple du psoas» dans un cours de danse. Comment, alors, trouver un point de consensus pour rendre le souffle littéraire d'une pensée, baignée dans l'univers du nord des États-Unis? Mes choix sont aussi guidés par ma subjectivité, par ce que j'ai appris d'un pays, d'une histoire. Je suis face à un énoncé qui parle directement au subconscient des gens dans la langue de départ, et c'est important qu'un peu de cet esprit soit transmis dans la traduction, même si c'est un livre pratique.

FC: Nous amenons notre point de vue d'éditeur et la volonté d'arriver à un texte qui soit le plus clair et le plus directement compréhensible possible. Dans une pratique qui fonctionne beaucoup par construction d'images et où le langage ajoute une autre couche d'images, comment le lecteur peut-il aller directement au cour de ce que cette pratique propose? C'est cet équilibre-là qui était en jeu dans nos dialogues de retours de traduction.

BA: C'est une question qui touche l'écriture même de l'auteur et qui est doublement posée par la traduction: à qui 
s'adresse-t-on? Et comment? La danse est aussi l'expression d'un savoir et d'un rapport du corps au langage qui est assez merveilleux. C'est très proche de ce que la poésie peut aller chercher en matière de ressources du langage et de palette d'adresses.

AF: Le langage des chorégraphes tient à un système de pensée, à un modèle culturel; si on les suit, on véhicule ce mode de pensée dans une nouvelle langue. Pour moi, la question s'est parfois posée: en tant que danseuse, je suis l'éponge de plusieurs systèmes culturels; en tant que traductrice vers le français, dois-je procéder à un transfert, «nettoyer», ou laisser passer en invoquant le caractère poreux de la langue?

BA: Poreux, mais à la limite de ce que permettent nos filtres culturels et de pensée. Nous avons notamment évoqué ensemble la différence entre la culture belge et la culture française: Florence et moi faisions remarquer qu'en Belgique, le fait d'intégrer ou d'entendre au quotidien aussi bien le français que le néerlandais modifie la manière dont on peut penser ou recevoir une autre langue que la sienne.

AF: Je me demandais si je devais accepter cette porosité et conserver des mots de la langue d'origine dans un texte qui se dit français. C'est la confrontation entre la théorie et le fait que la langue vit - et qu'elle danse, en l'occurrence.

Pourriez-vous donner un ou deux exemples où, côté éditeurs, vous auriez gardé un mot anglais, et où la traductrice aurait plutôt traduit?

BA: Le terme release technique a été un des enjeux présents du début à la fin. On avait d'abord adopté «technique release». Mais il y a eu la tentative de proposer une traduction...

AF : ... «détente anatomique»...

BA: ... ou «libération». On s'est rendu compte qu'il s'agissait d'une appellation propre, et c'est devenu l'objet d'une note de traduction. N'est-il pas important de conserver l'appellation originale s'il ne s'est pas dégagé parmi les praticiens une traduction qui s'impose d'elle-même? II faut en effet être sûr que le terme parle suffisamment au praticien. Et comme release technique circule d'une certaine manière dans le français, nous avons choisi de le garder.

FC: Depuis de nombreuses années, nous conservons les appellations dans leur langue d'origine. Nous cherchons à 
privilégier ce qui est le plus pertinent par rapport au monde de l'édition, de la danse, de la pratique, et tentons, autant que possible, d'être cohérents dans nos choix éditoriaux. C'est passionnant parce que, d'ouvrage en ouvrage, il y a toujours deux ou trois termes de vocabulaire qui cristallisent les discussions.

AF : J'ai d'abord traduit par «technique release»- car la danseuse en moi s'est formée en technique «release» à Berlin. Puis la traductrice en moi a pris le dessus: pourquoi ne pas traduire? C'était une question d'engagement déontologique: je ne pouvais pas associer mon nom à release technique, d'autant que depuis trois ans, je n'ai jamais entendu release technique dans le milieu francophone. S'est ensuivie une recherche de tous les mots possibles, sachant qu'il y aurait une perte et peut-être un glissement de sens.

BA: Pour abonder dans le sens de Florence, à chaque ouvrage, c'est comme si on décelait un certain nombre de termes spécifiques à un auteur ou à une pratique. On a une sorte de nuage de mots qui deviennent une matrice de la matière qui sera transmise. Et c'est de ce nuage dont on se sent responsable en tant qu'éditeur ou traducteur. Pour le livre de Nancy Topf, il était par exemple très complexe de maintenir la notion de deep, ou en tout cas de trouver une cohérence tout au long de l'ouvrage, pour que l'on puisse reconnaître un concept tout en élargissant son champ sémantique. Est-ce le même mot ou un autre? Comment procéder dans la traduction de tel ou tel passage, comment ce mot agit-il une fois qu'il est reçu? C'était là aussi un enjeu, avec d'un côté un ras-le-bol de la répétition, alors que la langue peut être plus riche, et de l'autre, l'idée qu'il faut aussi identifier le vocabulaire thématique structurant de la parole de l'autrice.

AF: Je parlerais même d'imaginaire et de sous-texte. Dans mon premier jet, je n'ai pas toujours traduit deep par "profond», "profondément», "profondeur», mais plutôt en fonction de l'environnement du mot. Dans le flux du texte, on pouvait tout à fait avoir "un endroit d'intimité» ou un mot éloigné de "profond». Puis vous avez voulu revenir à deepen dépit des répétitions.

BA: On s'est rendu compte de ce phénomène dans la pratique, où l'enseignant définit un vocabulaire propre, baigné 
dans le langage courant. Le mot prend tout son sens dans le cadre qui vient le porter - c'est l'adéquation entre un mot et son contexte. Est-ce qu'on maintient le mot, en laissant le contexte élargir son champ sémantique, ou est-ce que le mot va changer?

FC: C'est d'autant plus le cas pour les auteurs qui sont artistes et se permettent avec les mots un certain flou, une interprétation, une subjectivité. On y est confrontés dans la langue d'origine, et c'est décuplé dans la traduction.

AF: Le message reçu par une personne native met en jeu de nombreux éléments subliminaux qu'une personne non native doit pouvoir retrouver dans la version française. Chaque chorégraphe ou praticienne a sa manière d'être. Le vocabulaire de Nancy Stark Smith a quelque chose de très pastoral - le vocabulaire de l'Underscore m'évoque une grande prairie américaine. Il y a aussi la personnalité du praticien et une part d'image: le Body-Mind Centering ${ }^{\oplus}$ (BMC) convoque des images anatomiques, les fluides, les liquides, alors que le butô fait appel à un imaginaire plus poétique. II existe donc différentes approches, qui vont toucher les humains diversement, et elles sont à conserver.

BA: Quand nous traduisons un ouvrage, j'ai remarqué qu'il nous fallait en général au moins deux étapes de relecture et de discussion avec la traductrice pour que la matière puisse descendre plus bas que nos neurones, plus loin dans le corps, pour nous familiariser à la fois avec le contenu, la langue de l'auteur et la subjectivité du traducteur ou de la traductrice. Aude, qu'en est-il pour toi?

AF: II y a plusieurs étapes, à commencer par la lecture du texte, qui est déjà une traduction; c'est le moment de la réception, sensorielle, subjective en raison de mon vécu et de ce qui m'a formatée, et objective parce que j'ai une certaine connaissance de ce que tel mot veut dire dans le dictionnaire. Vient ensuite une reformulation globale interne, qui correspond au moment de compréhension, mais qui est aussi une interprétation personnelle. Ces premières étapes relèvent du domaine de la langue source. Puis il y a la déverbalisation: j'oublie les mots anglais pour transférer le message en français. C'est là que s'opère le transfert dans l'autre langue, par un changement du système de pensée et du modèle culturel. La reverbalisation dans la langue cible change en fonction 
des conditions de sortie (édition, sous-titrage...). Je produis un premier jet que je vais relire et peaufiner, en faisant appel à des experts si nécessaire. Pour l'ouvrage de Nancy Topf, j'ai travaillé avec une éducatrice BMC qui a l'habitude d'être au contact d'écritures. Pour la relecture, je fais parfois appel à mon père qui ne parle aucune autre langue que le français; il ne projette donc pas la connaissance d'une langue étrangère et me signale ce qu'il ne comprend pas. Très souvent, il pointe des passages que j'avais mal compris. Pour moi, c'est important qu'il n'y ait pas dans la tête du relecteur ou de la relectrice une comparaison avec l'original, mais que la relecture décèle les problèmes de français. Nous avons ensuite refait avec Baptiste et Florence une bonne partie de ce travail, à l'aune d'autres subjectivités et d'autres perceptions du texte, ce qui est déroutant.

FC: À la réception d'un texte, la lecture se fait d'office en français, et on retourne à l'anglais là où on a l'impression qu'il n'y a pas de connexion directe entre le mot et les phrases...

BA: ... ou avec la façon dont le mot agit. Je me souviens d'un passage à propos des yeux qui dit: Let them feel soft and heavy; imagine them sinking to the very bottom of their sockets. La première formulation d'Aude était «Détends les yeux et sens comme ils sont lourds. Imagine-les sombrer vers la pointe des orbites». "Sombrer» ne suscitait pas l'expérience physique qui me semblait correspondre au mot sink.

AF : Après demande de reformulation au plus près de l'original, j'ai proposé "lmagine-les s'enfoncer au fin fond des orbites».

BA: II y a eu une série de propositions: "couler», "s'écouler», «plonger», "se déverser», «tomber». On essayait de voir comment ce sink opérait physiquement, quelle sensation il allait développer, quelles actions il induisait au niveau notamment des yeux, de leurs orbites et du fond du crâne. Un même mot résonne différemment d'une personne à l'autre; nos subjectivités devaient donc trouver un consensus. Le verbe "couler», qui a finalement été retenu, conserve l'évocation du monde liquide et produit une expérience qui nous rassemble.

AF : Je n'avais pas du tout pensé à «couler». Nos discussions et le fait d'imaginer aussi ce qu'on entend en studio, "laisse couler», m'a amenée à considérer l'existence de ce terme 
- qui englobe le même univers que sink! C'est très enrichissant et formidable de confronter les subjectivités pour arriver à un choix.

BA: On ne publie qu'un ouvrage par an, ce qui nous demande de mesurer nos choix et nous permet d'accompagner le processus de traduction. On s'y investit beaucoup, on soutient et questionne le traducteur ou la traductrice. Cela peut être lourd si on n'est pas passionné par ce qu'on fait... On s'est donc entourés de passionnés!

Est-ce que, pour la traduction d'un texte de danse, la connaissance de la danse prime sur le style, ou le style sur la danse?

AF: C'était en négociation permanente: je me sentais responsable à la fois du souffle littéraire, du sous-texte et de la précision du mot. Chaque phrase a été vérifiée pour une restitution au plus près de l'original, avec parfois le constat qu' "on ne dirait pas comme ça en français». C'est un processus lourd, mais qui m'ouvre à d'autres manières de penser.

BA: Nombre de nos lecteurs ont probablement choisi le médium de la danse parce qu'ils ont moins d'affinité avec le verbe. Dans le processus de traduction, dans le choix des ouvrages que nous éditons, nous tentons de maintenir le lien avec la pratique de la danse et ses différents langages. Nous veillons à maintenir un ancrage parfois prosaïque, tout en gardant une qualité littéraire.

FC: Une traductrice ou un traducteur qui a des acquis en danse, c'est essentiel. On le perçoit très vite au cours de notre collaboration. On sent tout de suite le travail qu'Aude a fait autour des exercices. Pour nous, c'est primordial d'avoir ce point de vue. Nous avons eu des collaborateurs aux bagages et aux pratiques différents. En l'absence de connaissance de la pratique par la traductrice, l'auteur demande souvent qu'une personne de référence francophone relise la traduction.

Quelles sont les particularités du vocabulaire - peut-il y avoir polysémie?

AF: Contredanse a proposé un exercice que je trouve très intéressant: la traduction d'un même article par une praticienne et par une traductrice littéraire, qui pratique aussi, mais veut rendre ce qu'elle perçoit comme lyrique ou poétique, et qui sera prosaïque pour d'autres. Je pense à un cas pratique: comment traduire embodiment en danse? 
Mes études littéraires m'ont enseigné que l'équivalent est "incarnation», mais ce n'est pas le terme employé dans un studio de danse ou un cours de BMC.

FC: Nous avons en effet confié la traduction d'un même texte à deux traductrices ${ }^{11}$ et publié leurs deux traductions ${ }^{12}$ en y joignant une discussion sur la problématique de la traduction ${ }^{13}$.

AF : Et puis une publication correspond à un instant $t$. Dans l'article mentionné, Madie Boucon, une des traductrices, dit que six mois plus tard, elle aurait choisi "encorporation» et non plus «incorporation» comme elle l'avait fait. Le temps imparti fait qu'on n'a pas le cerveau élastique. J'aurais peutêtre pensé à certaines de vos propositions si j'avais eu un autre temps de traduction.

FC: Cela rend compte aussi d'un temps précis dans l'histoire et l'évolution de la danse. Par nos publications, nous construisons la culture de la danse en français. À la parution de Sentir, ressentir, agir, même si on s'était adjoint deux relectrices praticiennes du BMC, on nous a reproché d'avoir traduit certains termes propres à la pratique, alors que dans les studios, même dans l'école française, on utilise le vocabulaire anglais d'origine. Les choses évoluent. Dix ans plus tard, on ne traduit plus de la même manière, on ne parle plus de la même manière d'une pratique.

\section{Vos publications s'adressent à un public très large. Le desti- nataire de l'ouvrage influence-t-il aussi les choix de termes?}

FC: Nous voulons en effet nous adresser autant aux amateurs curieux qui ne connaissent pas du tout la pratique qu'à des spécialistes qui veulent utiliser nos ouvrages comme des références. Il faut trouver le vocabulaire le plus juste, pour être spécifique sans être abscons. Expliquer dans une note de traduction pourquoi on ne traduit pas un terme permet d'avoir une grille de lecture pour un public plus large. Quand on achoppe sur certains termes, on essaie de repérer dans

\footnotetext{
11. Bonnie Bainbrige Cohen, "The processus of embodiment", in De l'une à l'autre Composer, apprendre et partager en mouvements (coll.), 2010, p. 64.

12. "Le processus d'incorporation», traduit par Madie Boucon, et «Être-en-corps. "The processus of embodiment" ou comment être dans son corps", traduit par Élise Argaud, in De l'une à l'autre, p. 66-67.

13. "Conversations sur des enjeux de traduction avec Madie Boucon, Élise Argaud, Loulla Chourlin et Éric Zobel», in De l'une à l'autre, p. 68-72.
} 
ce qui a déjà été traduit ou écrit en français quels ont été les choix antérieurs, pourquoi et par qui ils ont été faits, afin de nous inscrire dans une culture existante. On essaie de croiser plusieurs critères.

BA: Et aussi de défendre le langage véhiculé par la danse, même si on tord parfois un peu le cou à la langue française ou à un entendement plus commun. Notre public premier reste néanmoins les danseurs et danseuses et les chorégraphes. II faut aussi qu'une phrase puisse se tenir et être efficace dans son adresse. Il y a en effet tout ce qui est particulier non pas au monde de la danse, mais à l'activité même du traducteur: refaçonner une phrase pour qu'elle trouve son existence dans la langue cible.

AF: C'est cette reformulation que je sous-entendais tout à I'heure par reverbalisation. La syntaxe reflète un système de pensée différent. En France, je suis les cours de danse contemporaine d'une personne originaire d'Argentine, dont le français est parfois approximatif, avec des «le» et des «la» au mauvais endroit, ce qui n'empêche pas de bien chorégraphier. La danse est vécue et transmise de nombreuses manières différentes, et le fait que la formulation ne soit pas entièrement correcte ne retire rien à sa qualité. Mais bien sûr, il y a des missions et un cadre éditorial à respecter!

Propos recueillis par Émilie Syssau.

\section{WwW.contredanse.org baptiste@ contredanse.org florence@ contredanse.org a.fondard.trad@gmail.com}

Baptiste Andrien, architecte de formation, conçoit et réalise plusieurs projets de films et d'installations, interrogeant les rapports du corps à l'espace. En 2001, il rencontre le travail de Lisa Nelson. II participe à plusieurs groupes de recherche sondant le Tuning Score de Lisa Nelson, dans des laboratoires de danse, des ateliers pédagogiques et des créations chorégraphiques. Depuis 2006, il collabore avec Florence Corin aux éditions et aux formations de I'association Contredanse. 
Après une formation d'architecte, Florence Corin s'oriente vers la création multimédia, principalement dans le champ de la danse et des technologies numériques. De 2004 à 2010, elle crée plusieurs installations, pièces chorégraphiques et interactives. Engagée depuis 1998 au sein de Contredanse, elle y est aujourd'hui coresponsable des publications. Depuis lors, elle y a réalisé, en collaboration avec Patricia Kuypers et maintenant avec Baptiste Andrien, la plupart des ouvrages publiés par la maison d'édition. Elle s'occupe également des formations.

Aude Fondard est traductrice littéraire, diplômée de l'Institut de traducteurs, d'interprètes et de relations internationales (ITI-RI, Strasbourg) en 2005 et certifiée par l'École de traduction littéraire (ETL/CNL, Paris) en 2019. Elle navigue au quotidien entre l'anglais, l'allemand, le grec moderne et le français. Elle s'est spécialisée dans la fiction féministe, les catalogues d'exposition, les écrits sur l'art vivant, la danse et les arts visuels et mène en parallèle une activité artistique fondée sur l'écriture créative et chorégraphique. 